\title{
Tratamiento informativo (framing) del candidato indepen- diente frente a los candidatos de partidos tradicionales en el estado de Nuevo León durante la campaña electoral 2015
}

Information treatment (framing) of the independent candidate versus traditional party candidates in the state of Nuevo León during the 2015 election campaign

\author{
Ana Carolina Flores Villalobos \\ Universidad Autónoma de Nuevo León \\ Orcid https://orcid.org/oooo-00o3-0652-9530 \\ anacaro.cg@hotmail.com
}

Resumen: Dentro de los procesos electorales los medios de comunicación juegan un importante papel y es esto uno de los aspectos que se demuestran en la presente investigación. El presente texto revisa cómo a través de los medios se da una cobertura mediática de la campaña electoral del 2015 en el estado de Nuevo León y cómo a partir de esta cobertura se logró posicionar a los candidatos a la gubernatura siendo el candidato independiente quien obtuvo la victoria, todo esto respaldado a partir de la teoría del framing la cual sustenta numerosos estudios en la línea de investigación de la comunicación política.

Palabras clave: Framing, campañas electorales, encuadre, medios de comunicación, comunicación política, candidatos independientes, agenda setting

\begin{abstract}
Within the electoral processes, the media play an important role and this is one of the aspects demonstrated in this research. This text reviews how media coverage of the 2015 electoral campaign in the state of Nuevo León was given through the media and how this coverage was used to position the candidates for the governorship, being the independent candidate the one who won the victory, all this supported by the framing theory which supports numerous studies in the research line of political communication.
\end{abstract}

Keywords: Electoral campaigns, framing, media, political communication, independent candidates, agenda setting

Fecha de recepción: 03/11/2021

Fecha de aprobación: 03/12/2021

Cómo citar este artículo: Flores Villalobos, A. C. (2021). Tratamiento informativo (framing) del candidato independiente frente a los candidatos de partidos tradicionales en el estado de Nuevo León durante la campaña electoral 2015. Revista de Comunicación Política, 3, e210302. https://doi.org/10.29105/rcp3-2 


\section{Introducción}

La naturaleza de las campañas estriba en las estrategias y objetivos que la condicionan y funcionan bajo dos premisas esenciales: persuadir e informar. Tienen como objetivo principal dar a conocer a un candidato, partido, ideología y establecer una vinculación entre gobernantes y votantes (Ahuactzin et al., 2014).

En el proceso electoral, la comunicación política es un elemento que se utiliza como estrategia fundamental para persuadir al ciudadano y al elector. Es el mejor instrumento con el que cuenta un candidato o partido político para posicionarse frente a sus electores. Cuando se habla de la relación entre política y comunicación, se hace referencia a una práctica entre ambas pudiéndose considerar principalmente su difusión masiva por medio de medios tradicionales, como la televisión, y los medios online y las redes sociales (Rospir, 2003).

Los medios de comunicación cumplen dos tipos de roles informativos: alertan al público acerca de los acontecimientos y cambios que están teniendo lugar en su entorno, cumpliendo así una función indicadora; pero también cumplen una labor como traductores de los lenguajes, es decir, hacen comprensibles las noticias para una gran parte del público que las consume (McCombs \& Evatt, 1995). Es necesario conocer el contenido real al que los ciudadanos son expuestos durante la campaña electoral cuando consumen los contenidos realizados por estos medios. En especial a través de la cobertura que hacen de la campaña, la cual será realizada con un tratamiento informativo, el cual pueda ser entendido por la ciudadanía a través del tratamiento informativo o framing que se ofrece (Muñiz, 2015).

Entman (1993) señala que la teoría del framing explica un proceso donde se seleccionan aspectos de la realidad misma los cuales se hacen resaltar en los medios de comunicación, para así difundir una problemática, acontecimiento, realidad, etc. Los frames pueden definirse como aquellos enfoques que se utilizan en determinado texto para dar una explicación y una forma a diversos asuntos o acontecimientos (Sádaba, 2001). Los periodistas van a tener como fin elegir qué es lo que se va a plasmar en el texto. Estos enfoques, también llamados encuadres, serán indispensables en el trabajo periodístico explicado desde la teoría del framing, constituyendo variables en el proceso noticioso y de creación de opiniones (Muñiz, 2015).

Lo dicho anteriormente permite asumir que existirán diversos encuadres respecto de un mismo acontecimiento y/o problema, asunto, etc. Se entiende así el framing como un proceso que conforma la creación, selección y establecimiento de ciertos encuadres noticiosos (Matthes, 2012). Los encuadres noticiosos son los que se van a encargar de establecer la forma en la cual va a ser organizada la información que se dará a conocer a los medios. Su utilización por parte de los periodistas conlleva una decisión sobre qué elementos que van a ser seleccionados, que es lo que se va a recalcar 
y que es lo que simplemente se va a omitir para así construir el discurso (Muñiz, 2011; Ryan, 2012; Scheufele, 2000).

En materia de campañas electorales, la legislación mexicana considera que los medios de comunicación deben dar una cobertura por igual a los partidos políticos. En el año 2014 se realizó una reforma política electoral a partir de la que se dio oportunidad de presentar candidaturas independientes. Por tanto, la cobertura de los medios de comunicación ya no debería ser exclusiva de partidos, sino también de este nuevo tipo de candidaturas. En el caso de las elecciones para gobernador del estado mexicano de Nuevo León del año 2015 surgió un candidato independiente. Así, Jaime Rodríguez Calderón “El Bronco” se postuló para obtener dicha gubernatura como independiente.

\section{La importancia de las campañas electorales}

Una campaña electoral es un conjunto de decisiones estratégicas utilizadas por los candidatos y partidos para lograr el voto de los ciudadanos. Tanto los candidatos como los partidos, así como los asesores de la campaña misma, analizan las acciones realizadas en el pasado para determinar estrategias a implementar en el presente y las visiones futuras, para así intentar convencer al votante de elegir al partido al cual representan (Martínez, 2008).

A partir de los cambios y las evoluciones en la década de los ochenta en América Latina, las estructuras económicas como políticas y la evolución tecnológica por la que se atravesó, se generó un nuevo núcleo social en el que la sociedad misma comienza a sustentarse en la información, la comunicación y el conocimiento, la cual a lo largo de los años sigue aún en un proceso de construcción el cual conlleva a la generación de nuevas formas de dar y recibir información, así como persuadir al receptor. Se espera que, dentro de este tipo de sociedad, la cual está basada en el conocimiento y la información, se pueda generar una serie de cambios en la manera de acceder, controlar, ejercer y producir el poder público. En este sentido, las campañas electorales han experimentado a lo largo del tiempo distintos cambios, tanto en su conceptualización, organización, financiamiento, así como en la forma de comunicación y la forma de movilizar al electorado (Valdez, 2010).

Estos procesos electorales no solo informan a la ciudadanía, también incentivan al voto. Son fruto de una necesidad, misma que se refiere a llegar al mayor número de electores. Tienen como objeto el dirigirse a un gran público y, de esta manera, no solo ser una actividad política, sino también una actividad social. La campaña electoral es, por tanto, el conjunto de actividades que coadyuvan a la configuración del voto y, por ende, a llevar a la conformación de los órganos representativos que se van a elegir (Soler, 2001).

Dentro de la política, las campañas son momentos en los cuales el candidato y/o partido va a exponer sus ideas, propuestas y van a estar en constante comunicación y contacto con la ciudadanía 
para tratar de convencer al elector con respecto a la aportación de su voto. No va a ser, por tanto, un momento cualquiera sino, por lo contrario, será un momento decisivo donde no solamente distintos partidos van a disputarse el voto sino, también es el tiempo en el cual se le va a dar legitimidad a las ideas y propuestas de estos, estableciéndolas así en la mente de los electores (Toussaint, 2000).

\section{Cobertura informativa de las campañas electorales}

Los procesos electorales constituyen un momento primordial en el desenvolvimiento de una sociedad, pues en ellas se hace presente el juego democrático. Constituyen un momento donde todos los sectores sociales se hacen presentes y ejercen la participación política, formándose una dinámica donde participan electores y candidatos. Dentro de esta dinámica es imposible que no se haga notar la presencia de los medios de comunicación, dándose un proceso de comunicación social fuerte de transmisión de información política hacia la ciudadanía (Aceves, 2000).

La comunicación que hoy se busca es una que sea capaz de lleguar al elector de manera individual, con atención personalizada para que atienda las inquietudes del votante y sus expectativas. Los medios de comunicación tienen la habilidad de cambiar la perspectiva y/o consideraciones de la opinión pública, los criterios que las audiencias tienen para evaluar a los candidatos y/o políticos o bien para tomar una decisión en cuanto a la emisión de su voto a determinado candidato, en su mayoría son influidos por la información dada por los medios de comunicación. Por ello, la información transmitida por los medios durante una campaña electoral sobre un tema en específico o bien sobre un candidato o partido es primordial en la decisión del votante y posteriormente en la emisión del voto (Valdez, 2010).

Los medios de comunicación configuran un espacio público a la acción y contexto político, pueden centrarse en procesos de comunicación política, primordialmente en los procesos electorales, tienen la capacidad de poder establecer una agenda política que permite desarrollar temáticas diversas como proyectos, propuestas y promesas de campaña, dar a conocer a los candidatos, etc. Por ello, esto los convierte en un instrumento para la construcción de la democracia, además que promocionan condiciones de competencia electoral más equitativa (Aceves, 2004).

La cobertura que ofrecen los medios de comunicación busca dar a conocer los acontecimientos electorales, abundando con ello en el conocimiento del candidato que tiene el ciudadano. La televisión, por su parte, es un medio que permite ver y escuchar al candidato, por lo que en una presentación en la televisión cada detalle deberá ser estudiado, tener definido dónde se hace la presentación, qué noticia se va a lanzar, quiénes acompañan al candidato, etc. La radio es otro de los medios de comunicación a considerar que, si bien tiene de manera explícita un gran poder, también se somete a considerables limitaciones en una campaña electoral. Su poder principal radica en el sentido de que 
es móvil, ya que puede ser escuchada en cualquier trayecto, llega a todos los sectores, incluso zonas rurales donde aún no es posible contar con televisión. Sin embargo, su limitante radica en que impide ver y, por lo tanto, percibir más cualidades personales del candidato (Lozano et al., 2012).

La presencia de los medios de comunicación dentro de las campañas electorales posibilita que una gran diversidad de mensajes se dé a conocer a los consumidores y audiencias de dichos medios. Se debe tomar en cuenta a qué mensajes y a qué contenidos están expuestos los receptores, que en este caso tienen la capacidad de convertirse además en electores. De esta manera, se pueden analizar y saber qué tan efectivos o no son dichos mensajes, su significado y el impacto que los mismos tienen en quien los recibe. Algo que ha sido estudiado desde la teoría del framing.

\section{Teoría del framing}

El origen de la teoría del framing surge en el contexto de la sociología interpretativa, asumiendo que las interpretaciones de la realidad que hacen las personas corresponden a un objetivo de análisis, definiendo una situación en particular. La teoría del framing da una aproximación a lo que sucede cuando se representa la realidad. Al hablar de definición de una situación, se hace referencia en las situaciones que son reales y que conllevan consigo consecuencias reales, de manera que al definirse por parte de los comunicadores e interpretarse por parte de la sociedad se llegue a una percepción de la realidad social, donde el individuo va a hacerse una idea de la situación tomando en cuenta para ello sus conocimiento, actitudes y experiencias, que en gran medida son establecidos o afectados por las representaciones de la realidad que hacen los medios (Sádaba, 2001).

La teoría del framing se utiliza en el estudio de los medios de comunicación para analizar y explicar cómo los periodistas interpretan las situaciones y realidades para darlas a conocer. Por ende, el framing va a ser un instrumento que permite describir un proceso y una interpretación de un significado en particular, siendo una herramienta para obtener significados de la realidad (Sadaba, 2001). Entman (1991) concibe el framing como una selección de la realidad, a través del que el periodista va a poner de manifiesto los acontecimientos y dar una determinada imagen de lo que está aconteciendo, de tal modo que, al seleccionar estos acontecimientos, los hará más notables ante la audiencia. El framing, por tanto, concibe la definición de la realidad como una cuestión de selección. De esta manera, lo que aparece en los medios parecerá que es lo que realmente está aconteciendo y casi siempre las noticias definirán la realidad tanto por lo que seleccionan como por lo que dejan de seleccionar.

Los frames son estructuras que permiten conocer la realidad, que trabajan señalando que la realidad se mira desde distintos lugares, distintas ventanas y que esa realidad cambia. Dichos frames, encuadres o marcos son instrumentos mentales que diferencian una cosa de otra, con los que se va a intentar dar una respuesta a lo que va a ser un fenómeno y que establecerán un marco que permitirá 
comprender el mensaje. Goffman (1986) parte de la sociología y utiliza también el concepto de "marco", señalando que los acontecimientos no solo se organizan en la mente, sino en la sociedad misma. Indica que las definiciones de una determinada situación van a construirse de acuerdo con una organización de principios dentro de acontecimientos. Cada persona va a definir las situaciones a partir de los frames, mismos que darán sentido a los acontecimientos.

Tal y como señalan Igartua et al. (2005), el proceso del framing está relacionado tanto con la selección y el énfasis dado en el texto informativo a ciertas palabras, expresiones e imágenes para contextualizar una realidad y concretar una perspectiva dentro de la información. El framing se puede trasladar a distintos niveles en el proceso de información, por lo que quien se adentra en dicho proceso informativo, va a poder enfocar o encuadrar la información desde diferentes puntos de vista (Gimenez 2006). En este sentido, el frame puede entenderse como una idea organizadora de los contenidos informativos que va a contener una noticia. Dicha idea va a dar un contexto y a su vez va a sugerir de que se trata determinado tema, a partir de una selección y un análisis de este (Tankard, 1991). Por otra parte, Gitlin (1980) señala que los frames son recursos que utilizan los medios desde una posición la cual predomina, esto con el fin de organizar la realidad de una sociedad. Los frames de los medios van a situarse por tanto en un dominio cognoscitivo y van a ir ligados hacia una actitud informativa, teniendo así una visión constructivista de la realidad (Sádaba, 2001)

El uso de la teoría del framing en la investigación en comunicación, Sádaba señala que ha tenido un desarrollo notable, sobre todo porque se presentan debates referentes a los medios de comunicación que tienen que ver con la posibilidad que tiene el periodista de utilizar el framing para dar a conocer la realidad objetivamente y/o tal y como es. Considerándose, por tanto, como una práctica periodística con actitud informativa, ética y objetiva. En el área del periodismo, la teoría del framing se fundamenta en que los medios de comunicación juegan un papel mediador entre el hombre y la sociedad, donde se van a dar a conocer las noticias. Los medios, por tanto, van a situarse como intermediarios entre el mundo exterior y la audiencia, siendo los medios en la mayoría de las ocasiones el único modo de acceso de lo que está sucediendo (Sádaba, 2001).

Tal y como señala Meyer (2014), la cobertura que brindan los medios de comunicación como prensa escrita y televisión a las campañas electorales se encuadra en términos de juego estratégico (strategic game frame), lo que permite que los candidatos tengan importancia, así como presencia en los medios esto con el fin de obtener mayor beneficio electoral. Esto ayuda a que los medios de comunicación puedan llevar a cabo un tratamiento de asunto o temático (issue frame), el cual a su vez va a ayudar al debate sobre los candidatos, sus propuestas y temáticas de índole político (de Vreese, 2012; Cappella \& Jamieson, 1997). Si bien es el tratamiento de juego estratégico el que resulta ser dominante, ambos frames (juego estratégico y temático) se confrontan haciéndose presente en las noticias la crítica, conflicto y el debate (Muñiz \& Ramírez, 2014). Así, los medios son los que crean un significado de lo que ocurre en la sociedad (Sádaba, 2001). 
En lo que respecta a la cobertura de campañas electorales realizadas por los medios de comunicación, se realizaron una serie de trabajos donde se tenía como objetivo identificar la equidad de la cobertura a los diferentes candidatos. Sin embargo, en su mayoría se llega a la conclusión de que los medios de comunicación dan una cobertura no equitativa o más bien prioritaria hacia un candidato en particular, que es el que pertenece al partido que tiene el poder (Aceves, 2000; Martínez \& Godínez, 2013). El framing del asunto o temático (issue frame) es el que va a permitir aportar información acerca de las propuestas de campaña, las problemáticas que acontecen y las soluciones para resolverlas, así como las consecuencias de estas, con ello el ciudadano y/o votante tiene el acceso a información que permite conocer la posición política de los candidatos, así como también sus propuestas. El frame del asunto o temático va a permitir determinar como bien lo dice el concepto, las temáticas políticas dentro del proceso de una campaña electoral (Muñiz 2015). Por otra parte, el encuadre de estrategia (strategy frame), es aquel que coadyuva a que el candidato y/o partido político lleve a cabo estrategias las cuales lo posicionen con una intención de voto favorable, a su vez se presenta el encuadre de juego (game frame) en el cual se expone que los medios de comunicación presentan a las noticias como un juego en el que los partidos y los políticos compiten por tener una posición favorable ante el ciudadano.

Por su parte, Lozano et al. (2012) detallan que los medios informativos nacionales mexicanos, dedican la cobertura de las campañas electorales en gran parte al desprestigio, los ataques, las descalificaciones, las personalidades ya sean positivas o negativas y la posición en la que están, no todos los candidatos, sino los dos más fuertes, además de que no se centran en lo que realmente es relevante: las propuestas. Esta descripción del tipo de cobertura de las campañas electorales sitúa a dichos estudios cerca de los planteamientos realizados desde la teoría del framing acerca del tratamiento informativo de la política y, más en específico, de las campañas electorales (Muñiz, 2015).

Una de las líneas de estudio desde la teoría del framing es la que se centra en el análisis de los encuadres noticiosos que se presentan en las noticias de los medios dedicadas a la política. Dentro de dichas noticias se centra la importancia de la información que se da sobre política, en especial durante las épocas de campañas electorales, donde tiende a aumentar el debate de los medios de comunicación en torno al tema. Ello tiene como objetivo el determinar una relación existente entre las etapas de la vida política y el desarrollo de las actitudes políticas (Muñiz, 2015; Schuck et al., 2013).

En el entorno político, sobre todo en momentos electorales, se intensifica el trabajo de las élites y candidatos para tratar de introducir sus encuadres en el entramado informativo de los medios con el objetivo de influir en los comportamientos de los ciudadanos (Hanggli \& Kriesi, 2012; Muñiz, 2015). La élite política por tanto, traslada a los medios en ciertos encuadres (advocacy frames) esto con el fin de expresar sus puntos de vista sobre lo acontecido actualmente, a su vez, en los medios también se presentan encuadres periodísticos (journalistic frames), los cuales son utilizados por los 
periodistas para dar un enfoque a la información que transmiten los actores políticos pudiendo mantener el encuadre original, proponer un encuadre diferente o bien dar uno totalmente opuesto al del político, realizando una interpretación (Muñiz, 2015; de Vreese, 2012).

\section{El contexto electoral en Nuevo León en 2015}

Durante el año 2015 se realizaron diversas elecciones en el territorio mexicano, entre ellas, las elecciones para gobernador de varios estados, como el caso de Nuevo León. Entre las candidaturas para obtener la gubernatura en distintos estados, es precisamente el estado de Nuevo León donde por primera vez se presentan las candidaturas independientes, en el marco de este proceso electoral de 2015. Básicamente, la reforma electoral 2013-2014 incluyó la posibilidad de las candidaturas independientes para competir por cargos de elección popular (Berumen \& Medellín, 2016). Entre sus diversas modificaciones y adiciones a la ley se hacen presentes diversas disposiciones para los candidatos independientes. En la Ley General de Instituciones y Procesos electorales (Cámara de Diputados del H. Congreso de La Unión, 2020), se encuentran disposiciones de diversos rubros, entre ellos las candidaturas independientes. Incluyendo modificaciones en el financiamiento, el acceso a los medios de comunicación, franquicias postales, propaganda, etc. Dichas candidaturas están incluidas con las candidaturas de partidos tradicionales.

En el caso particular de Nuevo León, la candidatura independiente a gobernador fue la presentada por Jaime Rodríguez Calderón "El Bronco". La estructura de apoyo de dicho candidato capitalizó el uso de las redes sociales, logrando un total de 394182 firmas de apoyo a su candidatura electoral. Finalmente, en marzo del 2015 se hizo legal su registro como candidato independiente a la gubernatura del estado de Nuevo León (Berumen \& Medellín, 2016).

A pesar de que la Reforma Política Electoral 2013-2014 planteaba un proceso electoral justo y equitativo para todos los candidatos en cuanto a su acceso a los medios, en lo que respecta a la campaña electoral, el candidato independiente a la gubernatura del estado de Nuevo León Jaime Rodríguez Calderón "El Bronco" prefirió priorizar el uso de redes sociales para establecer su estrategia de campaña respecto a la contienda por la gubernatura del estado de Nuevo León (Berumen \& Medellín, 2016).

A partir de los datos oficiales de la Comisión Estatal Electoral y el Tribunal Electoral del Poder Judicial de la Federación (TEPJF), Berumen y Medellín (2016) señalan que a Jaime Rodríguez Calderón le correspondió un apoyo oficial para hacer campaña 383,329.46 pesos (Comisión Estatal Electoral, 2015, p. 34), es decir, menos de 1\% de los topes de campaña referido a los partidos políticos. Debido a esto, el candidato independiente recurrió principalmente al financiamiento privado, que le fue reconocido como legal por el órgano electoral a casi unos días de la fecha de la elección (7 de junio), siempre con un tope de hasta 49,929,949.27 pesos (Acuerdo CEE, 2015). Finalmente, Jaime 
Rodríguez Calderón utilizó en su campaña, de acuerdo con la Unidad Técnica de Fiscalización del Instituto Nacional Electoral (INE), el monto de 8,907,441.01 pesos, cifra validada en la resolución definitiva del Tribunal Electoral del Poder Judicial de la Federación (TEPJF). Una cantidad no comparable con la manejada por los candidatos oponentes: Ivonne Álvarez, 42.3 millones de pesos, y Felipe de Jesús Cantú, 39.4 millones de pesos, gastos reconocidos formalmente ante la Unidad Técnica de Fiscalización del INE.

La reforma a la legislación electoral fue la que permitió a Jaime Rodríguez Calderón "El Bronco", tener la posibilidad de inscribirse en dicho proceso electoral y posteriormente ganar la gubernatura en el estado de Nuevo León. Finalmente, la votación del 7 de junio se resolvió a favor del candidato independiente con una considerable ventaja sobre sus opositores. La votación reconocida por el órgano electoral fue la siguiente: Felipe de Jesús Cantú 466543 votos (22.32 \%); Ivonne Álvarez 498644 (23.85 \%) y Jaime Rodríguez Calderón 1020552 votos (48.82 \%) (Comisión Estatal Electoral, 2015).

\section{Metodología}

En la presente investigación se llevó a cabo un diseño de estudio cuantitativo, no experimental transversal y asociativo, gracias al cual se puede determinar si dos variables están correlacionadas o no; lo que permite conocer si un aumento o disminución en una variable coincide un aumento o disminución en otra. También permite comparar las diferencias existentes en ciertas variables en función de los grupos de las variables que se utilice como independiente. Para obtener los datos se realizó un estudio de análisis de contenido, siendo analizados los mensajes de televisión y prensa escrita publicados durante la campaña electoral de Nuevo León de 2015.

El análisis de contenido es una técnica de investigación que se utiliza para analizar los mensajes que la audiencia que la consume está recibiendo. Es una técnica que tiene un conjunto de procedimientos que permiten interpretar "productos comunicativos" (mensajes, textos o discursos), los cuales proceden de procesos específicos de comunicación previamente registrados, y que, basados en técnicas de medida, a veces cuantitativas (estadísticas basadas en el recuento de unidades), a veces cualitativas (lógicas basadas en la combinación de categorías) van a tener como fin el crear y procesar datos relevantes acerca de las distintas condiciones en que se ha producido y ejemplificado la información que llega a los receptores (Marañón, 2015, p. 132).

Las unidades de análisis, en este caso noticias, fueron seleccionadas a partir de determinadas características; debido a que la temática que se aborda es acerca del tratamiento informativo (framing) del candidato independiente frente a los candidatos de partidos tradicionales en el estado de Nuevo León durante la campaña electoral 2015, fueron tomadas en cuenta únicamente las noticias tanto de prensa escrita como de televisión referidas a la campaña electoral de los candidatos Jaime 
Rodríguez Calderón “El Bronco”, Ivonne Liliana Álvarez García y Felipe de Jesús Cantú Hinojosa. Cabe mencionar que dentro de la campaña electoral 2015 a la gubernatura del Estado de Nuevo León se presentaron otros candidatos a la misma, sin embargo, se decidió tomar en cuenta únicamente al candidato independiente y a los candidatos de partidos tradicionales (en este caso PRI y PAN) y de esta manera determinar el impacto que las mismas tuvieron durante el periodo de elecciones del año correspondiente.

Las unidades de contexto utilizadas correspondieron a la prensa escrita y a la televisión. En prensa escrita, los periódicos que fueron analizados para la presente investigación fueron $A B C$ de Monterrey, El Horizonte, El Norte, El Porvenir, Milenio MTY, Publimetro y Reporte Índigo. En televisión, las noticias analizadas fueron las emitidas por Televisa, Multimedios y TV Azteca.

El muestreo corresponde al periodo de campaña electoral 2015 por la gubernatura del estado de Nuevo León, siendo éste el período de elecciones electo para la realización del estudio. La muestra seleccionada se tomó en cuenta a partir del día 6 de marzo de 2015 hasta el día 3 de junio de 2015, siendo seleccionadas las noticias de prensa escrita correspondientes a ese período. Las noticias seleccionadas en televisión fueron las correspondientes a los horarios de 7:00 am a 2:00 pm y el otro horario de selección corresponde al de 6:00 pm a 12:00 am. En televisión se hizo el proceso de selección en los horarios mencionados con el propósito de que durante el horario de 3:00 pm a 5:00 pm se generara la actualización de noticias correspondientes a los candidatos analizados.

El tamaño de la muestra fue de un total de 430 noticias, específicamente en televisión contó con una submuestra de 237 noticias, mientras que de prensa escrita se contó con un total de 193 notas periodísticas.

El instrumento utilizado para realizar el análisis de las noticias de la presente investigación consistió en dos libros de códigos, uno de prensa y otro de televisión, los cuales contienen variables que ayudaron a obtener información concreta sobre el contenido de las noticias analizadas. Para ello, fue necesario realizar secciones de este, las cuales permitieron obtener información más precisa acerca de que tratamiento se les dio a los tres candidatos analizados (en este caso Jaime Rodríguez, Felipe de Jesús Hinojosa e Ivonne Álvarez). Dichas secciones permitieron determinar aspectos como el tiempo estimado que los candidatos tuvieron ante los medios de comunicación, el carácter informativo con el cual eran dados a conocer, la extensión de las notas, si daba o no presencia a los candidatos, si eran o no protagonistas de ésta, etc.

Las variables que fueron estudiadas en el estudio fueron:

\section{Presencia del Candidato}

Se evaluó si cada candidato tuvo presencia en la noticia como candidato principal. En este caso, se analizaron únicamente las noticias donde aparecen el candidato independiente, el candidato del PAN y la candidata del PRI. 


\section{Valencia afectiva de las noticias}

Además, también se evaluó si la noticia sobre cada candidato se presentó con un carácter negativo $(\mathrm{o}=\mathrm{No} ; 1=\mathrm{S} 1$ ) y positivo $(\mathrm{o}=\mathrm{No} ; 1$ = Sí) para cada uno de los candidatos analizados. Esta variable permite determinar la valencia afectiva que se le dio a cada candidato dentro de los medios de comunicación analizados, tanto prensa escrita como televisión.

\section{Framing episódico y temático de la información}

Para cada noticia se evaluó si la misma proporcionaba una información amplia, discutía razones, fuentes, responsabilidades de gobierno o empresas etc., o por el contrario proporcionaba información breve y concreta sin relacionar e involucrar causas, fuentes, etc. La variable, además de proporcionar información acerca de un tema abordado, permite determinar la extensión de la noticia proporcionando el nivel de cobertura.

\section{Framing del acontecimiento narrado}

Se evaluó la presencia en las noticias del encuadre de conflicto, a partir de la propuesta realizada originalmente por Semetko y Valkenburg (2000) y en la versión al español usada, entre otros, por Muñiz (2011). En concreto, se codificó mediante una escala de tres reactivos si en el relato se aludía o se hacía referencia o no a "cierto desacuerdo entre partidos políticos, individuos, grupos, instituciones o países", a "dos o más posturas diferentes en torno al tema o problema abordado" o a que "un partido político, individuo, grupo, institución o país realiza algún tipo de reproche a otro partido político, individuo, grupo, institución o país". La consistencia interna de la escala fue alta tanto para televisión $(\alpha=.74)$ como para prensa $(\alpha=.75)$, con valores similares a los obtenidos en otros estudios previos (Dimitrova \& Strömbäck, 2012; Semetko \& Valkenburg, 2000).

En contraposición a la existencia de conflicto, que marca la presencia en la nota de información relativa a posturas encontradas e incluso enfrentamiento entre actores, se consideró la posible existencia de un encuadre que diera a conocer la existencia de debates sobre propuestas que derivaran en acuerdos políticos. En este sentido, en el presente estudio se trabajó con una escala compuesta por cuatro reactivos que evaluaban si en el relato se aludía o se hacía referencia o no a los siguientes aspectos: "el texto enfatiza el debate entre actores políticos acerca de un tema o asunto concreto", "el texto presenta la toma de decisiones políticas como un acuerdo entre actores", "el texto enfatiza el acuerdo logrado por los actores tras una negociación alrededor de la decisión informada" y "el texto presenta la toma de decisiones políticas como escucharse mutuamente, como comprensión mutua, etc.”. El análisis factorial realizado con los cuatro ítems convergió en un único componente, tanto para la televisión, $\mathrm{KMO}=.756, \chi^{2}(6, N=351)=567.140, p<.001$, como para la prensa, $\mathrm{KMO}=.647$, $\chi^{2}(6, N=304)=383.784, p<.001$. La escala, además, presentó buena consistencia interna en la televisión $(\alpha=.79)$ y aceptable en la prensa $(\alpha=.67)$. 


\section{Framing político de los acontecimientos}

Tomando en cuenta los estudios previos existentes (Aalberg et al., 2012; Cappella \& Jamieson, 1997; Dimitrova \& Strömbäck, 2012; Elenbaas \& de Vreese, 2008; Muñiz, 2015), se utilizó una escala de cinco reactivos para medir la presencia del encuadre de juego estratégico (strategic-game frame) dentro de las noticias. Se evaluó si la nota señalaba, mencionaba o utilizaba (1) o no (o) aspectos como "metáforas generalmente asociadas con el deporte, competencia o incluso la guerra" o si "aporta datos de opinión, encuestas y/o posición de la opinión pública y ciudadanía hacia los políticos, partidos, campaña electoral, asuntos, etc.”. La consistencia interna de la escala fue baja tanto para televisión ( $\alpha=.52)$ como para prensa $(\alpha=.53)$, pero en consonancia con estudios previos (e.g. Muñiz, 2015).

\section{Resultados}

\section{Análisis de las variables descriptivas}

De acuerdo con las 430 noticias que se analizaron, la presencia de los candidatos a la gubernatura del estado de Nuevo León dentro de las mismas fueron variantes. Dentro de la variable del candidato se determinó no solo el número de ocasiones en las que el candidato se mencionó dentro de una noticia, sino también en que fue protagonista o bien candidato principal de la nota. En este caso, Ivonne Álvarez (PRI) protagonizó el 25.35\% en noticias en televisión, mientras que Felipe Cantú (PAN) obtuvo un total del 20.22\% y Jaime Rodríguez por último se presentó ante televisión como candidato principal con un $21.93 \%$ en noticias. En lo que a prensa escrita respecta, Ivonne Álvarez (PRI) protagonizo un total del 27.3\% de las notas, Felipe Cantú (PAN) obtuvo un 19.1\% y por último Jaime Rodríguez (Independiente) con un $\mathbf{1 7 . 1 \%}$ de apariciones en las notas periodísticas. El estudio arrojó una significancia de $\mathrm{p}=.390$ lo que indica que no se presentaron diferencias significativas entre los medios (prensa escrita y televisión), $\chi 2(2, \mathrm{~N}=430)=1.882, \mathrm{p}=.390$. (Ver Tabla 1).

Tabla 1. Candidato principal de la nota

\begin{tabular}{ccccc}
\hline \multirow{2}{*}{ Medio } & \multicolumn{2}{c}{ Televisión } & \multicolumn{2}{c}{ Prensa } \\
& $N$ & $\%$ & $N$ & $\%$ \\
\hline Ivonne Álvarez & 89 & 25.35 & 83 & 27.3 \\
\hline Felipe Cantú & 71 & 20.22 & 58 & 19.1 \\
\hline Jaime Rodríguez & 77 & 21.93 & 52 & 17.1 \\
\hline
\end{tabular}




\section{Carácter evaluativo del candidato}

La prueba $t$ de Student realizada al candidato Felipe de Jesús Cantú arrojó diferencias estadísticamente significativas en cuando al carácter evaluativo utilizado para dar cobertura mediática a su actividad, $t(344)=-3.307, p<.001$. Se observa cómo la prensa presentó un carácter evaluativo más favorable del candidato $(M=.25, D E=.46)$ respecto de la televisión $(M=.12, D E=.34)$. Con esto puede demostrarse que ambos medios de comunicación (prensa y televisión escrita) ofrecieron un tratamiento informativo distinto, esto en el caso de Felipe de Jesús.

Esto a diferencia de la candidata del Partido Revolucionario Institucional, Ivonne Álvarez, para quien no fueron estadísticamente significativas las diferencias entre los medios, $t(353)=-0.752$, $p=.453$. En todo caso, se puede observar que al igual que con Felipe de Jesús Cantú la prensa presentó un carácter evaluativo más favorable $(M=.21, D E=.59)$ con respecto a la televisión $(M=.17$, $D E=.45$ ). En el caso de la candidata Alianza por tu Seguridad se pudo observar cómo ambos medios de comunicación presentan distintos resultados ofreciendo la prensa escrita un tratamiento informativo más favorable (Ver Tabla 2)

Por último, las estadísticas de Jaime Rodríguez Calderón presentaron diferencias estadísticamente significativas entre ambos medios de comunicación, $t(428)=-2.696, p=.007$. Presentando carácter evaluativo desfavorable en televisión $(M=-.15, D E=.45)$ respecto a la prensa escrita, en la cual también arrojó carácter evaluativo desfavorable $(M=-.03, D E=.51)$.

Con Jaime Rodríguez Calderón se puede observar que, al contrario de Ivonne Liliana Álvarez y Felipe de Jesús Cantú, el tratamiento informativo que ofrecieron ambos medios fue similar, en este caso desfavorable para el candidato independiente por la gubernatura del estado de Nuevo León. Estos resultados permiten observar que la valencia afectiva informativa que los medios brindaron a Jaime Rodríguez fue distinta a la ofrecida a Ivonne Álvarez y Felipe de Jesús (Ver Tabla 2).

Tabla 2. Carácter evaluativo del candidato

\begin{tabular}{cccc}
\hline Candidato & Medio de comunicación & M & DE \\
\hline \multirow{2}{*}{ Felipe de Jesús Cantú } & Televisión & .12 & .34 \\
& Prensa & .25 & .46 \\
\hline \multirow{2}{*}{$\begin{array}{c}\text { Ivonne Liliana Álvarez } \\
\text { Jaime Rodríguez Cal- } \\
\text { derón }\end{array}$} & Televisión & .17 & .45 \\
& Prensa & .21 & .59 \\
\hline
\end{tabular}




\section{Uso de los diferentes encuadres en los medios}

Dentro del análisis de las noticias otra de las variables que se presentaron fue la de framing episódico y temático de la información. La televisión obtuvo un total de 13.9\% de noticias donde se proporcionaba un contexto amplio del hecho y un total del $86.1 \%$ donde la noticia informaba sobre un hecho en concreto. En cuanto a la prensa escrita, se obtuvo un total de 10.9\% de notas con noticias que proporcionaban un amplio contexto mientras que la cobertura de hechos concretos se obtuvo un total de 89.1\%de notas periodísticas. El estudio determinó que no se encontraron diferencias significativas entre los medios en la utilización del framing episódico y temático de la información, $\chi^{2}(1, N$ = 430) $=0.897, p=.344($ Ver Tabla 3$)$.

Tabla 3. Framing episódico y temático de la información

\begin{tabular}{|c|c|c|c|c|}
\hline & & \multicolumn{2}{|c|}{ Medio de Comunicación } & \multirow{2}{*}{ Total } \\
\hline & & Televisión & Prensa & \\
\hline \multirow{2}{*}{$\begin{array}{l}\text { La nota proporciona el } \\
\text { contexto más amplio del } \\
\text { evento }\end{array}$} & Frecuencia & 33 & 21 & 54 \\
\hline & Porcentaje & 13.9 & 10.9 & 12.6 \\
\hline \multirow{2}{*}{$\begin{array}{l}\text { La nota sólo informa del } \\
\text { hecho concreto }\end{array}$} & Frecuencia & 204 & 172 & 376 \\
\hline & Porcentaje & 86.1 & 89.1 & 87.4 \\
\hline \multirow{2}{*}{ Total } & Frecuencia & 237 & 193 & 430 \\
\hline & Porcentaje & 100 & 100 & 100 \\
\hline
\end{tabular}

La prueba $t$ de Student realizada con respecto al encuadre de juego estratégico arrojó diferencias estadísticamente significativas en cuando su presencia dentro de la cobertura mediática de la campaña, $t(389)=5.342, p<.001$. Se observa cómo la televisión uso más este encuadre $(M=.23, D E$ $=.28)$ respecto de la prensa $(M=.11, D E=.16)$. Lo que permite comprobar que se presentaron diferencias en el tratamiento informativo en ambos medios de comunicación. En el encuadre de asunto el estudio no arrojó diferencias estadísticamente significativas entre los medios, $t(428)=-1.415, p=$ .158 , detectándose en la televisión una presencia del encuadre $(M=.19, D E=.22)$ igual con respecto a la prensa $(M=.16, D E=.19)$. Tal y como se puede observar anteriormente, en el encuadre juego estratégico la televisión hace más uso del presente encuadre con respecto a la prensa. 
Tabla 4. Encuadres en medios de comunicación

\begin{tabular}{lccc}
\hline & Medio de comunicación & $M$ & $D E$ \\
\hline $\begin{array}{c}\text { Encuadre de juego es- } \\
\text { tratégico }\end{array}$ & Televisión & .23 & .28 \\
Encuadre de asunto & Prensa & .11 & .16 \\
& Televisión & .19 & .22 \\
Encuadre de conflicto & Prensa & .16 & .19 \\
& Televisión & .11 & .22 \\
Encuadre de debate & Prensa & .14 & .22 \\
político & Televisión & .05 & .16 \\
& Prensa & .04 & .14 \\
\hline
\end{tabular}

Lo mismo sucedió para el uso del encuadre de conflicto, $t(428)=-1.430, p=.153$, el cual tampoco presenta diferencias estadísticamente significativas, teniendo la prensa una utilización del encuadre de conflicto $(M=.14, D E=.22)$ igual que la televisión $(M=.11, D E=.22)$. A diferencia de los encuadres anteriores, en esta ocasión es la prensa escrita el medio de comunicación que tendía a utilizar más este encuadre. Por último, para el encuadre de debate no se encontraron diferencias estadísticas significativas, $t(428)=0.341, p=.733$, presentando la televisión el mencionado encuadre $(M=.05, D E=.16)$ en un nivel igual a la prensa $(M=.04, D E=.14)$. Los resultados mencionados permiten observar que, aunque las estadísticas no fueron estadísticamente significativas ambos medios de comunicación (televisión y prensa escrita) utilizaron variación en los encuadres presentados dentro de las noticias, lo cual demuestra que el tratamiento informativo ofrecido fue distinto en noticias de televisión frente a las de prensa escrita (Ver Tabla 4).

\section{Uso de los diferentes encuadres de los candidatos}

La prueba ANOVA realizada con respecto al encuadre de juego estratégico no arrojó diferencias estadísticamente significativas en cuando su presencia dentro de la cobertura donde cada candidato fue protagonista, $F(2,427)=0.333, p=.717$. Por tanto, se observa que en términos generales todos los candidatos presentaron una presencia similar en noticias enfocadas con el encuadre de juego estratégico siendo Jaime Rodríguez Calderón quien presentaba ligeramente más este encuadre $(M=.19, D E=.25)$, siguiendo Felipe de Jesús Cantú $(M=.18, D E=.24)$ y por último Ivonne Álvarez $(M=.16, D E=.23)$ quien menos presento el encuadre en sus noticias (Ver Tabla 5$)$. 
Tabla 5. Encuadres en los candidatos

\begin{tabular}{|c|c|c|c|}
\hline & Candidato & $M$ & $D E$ \\
\hline \multirow{4}{*}{$\begin{array}{l}\text { Encuadre de juego es- } \\
\text { tratégico }\end{array}$} & Felipe de Jesús Cantú Rodríguez (PAN) & .18 & .24 \\
\hline & $\begin{array}{c}\text { Ivonne Liliana Álvarez García (Alianza por tu } \\
\text { seguridad) }\end{array}$ & .16 & .23 \\
\hline & Jaime Rodríguez Calderón el Bronco & .19 & .25 \\
\hline & Total & .17 & .24 \\
\hline \multirow{4}{*}{ Encuadre de asunto } & Felipe de Jesús Cantú Rodríguez (PAN) & .17 & .21 \\
\hline & $\begin{array}{c}\text { Ivonne Liliana Álvarez García (Alianza por tu } \\
\text { seguridad) }\end{array}$ & .19 & .21 \\
\hline & Jaime Rodríguez Calderón el Bronco & .15 & .20 \\
\hline & Total & .17 & .21 \\
\hline \multirow{4}{*}{ Encuadre de conflicto } & Felipe de Jesús Cantú Rodríguez (PAN) & .19 & .21 \\
\hline & $\begin{array}{c}\text { Ivonne Liliana Álvarez García (Alianza por tu } \\
\text { seguridad) }\end{array}$ & .15 & .20 \\
\hline & Jaime Rodríguez Calderón el Bronco & .17 & .21 \\
\hline & Total & .12 & .22 \\
\hline \multirow{4}{*}{$\begin{array}{l}\text { Encuadre de debate } \\
\text { político }\end{array}$} & Felipe de Jesús Cantú Rodríguez (PAN) & .04 & .15 \\
\hline & $\begin{array}{c}\text { Ivonne Liliana Álvarez García (Alianza por tu } \\
\text { seguridad) }\end{array}$ & .03 & .11 \\
\hline & Jaime Rodríguez Calderón el Bronco & .07 & .19 \\
\hline & Total & .05 & .15 \\
\hline
\end{tabular}

En el caso del encuadre de asunto, no fueron estadísticamente significativas entre los diferentes candidatos, $F(2,427)=1.726, p=.179$, siendo Ivonne Álvarez quien predominaba en el mencionado encuadre $(M=.19, D E=.21)$, seguido de Felipe de Jesús $(M=.17, D E=.21)$. Por último, Jaime Rodríguez fue quien menos presentó este encuadre de asunto $(M=.15, D E=.20)$. A pesar de que los resultados no son estadísticamente significativos, se puede observar que Jaime Rodríguez es quien menos presenta este encuadre, lo cual hace ver que hay una diferenciación del tratamiento informativo ofrecido al independiente frente a los candidatos del PRI y PAN (Ver Tabla 5).

Sucedió lo mismo en el encuadre de debate en el cual no se arrojan diferencias estadísticamente significativas, $F(2,427)=2.414, p=.091$. Con todo, Jaime Rodríguez fue quien más presentó este encuadre $(M=.07, D E=.19)$, seguido de Felipe de Jesús, $(M=.04, D E=.15)$ y por último Ivonne Álvarez fue quien menos presentó noticias con frame de debate $(M=.03, D E=.11)$. Sin embargo, en el uso del encuadre de conflicto, sí se presentan diferencias estadísticas significativas $F(2,427)=$ 
$13.551, p<$.oo1. Aquí, se observa que Jaime fue el que más se presentó con este encuadre de conflicto $(M=.20, D E=.27)$, mientras que Felipe fue el segundo que más presentó este encuadre en su cobertura $(M=.12, D E=.22)$ e Ivonne la que menos estuvo presente en noticias con frame de conflicto ( $M$ $=.07, D E=.15)$.

Ambos medios de comunicación ofrecieron un tratamiento informativo con más presencia de encuadre de debate y de conflicto a Jaime Rodríguez Calderón, por lo que una vez más y con base a los resultados obtenidos puede observarse que los medios ofrecieron un tratamiento informativo distinto a Jaime Rodríguez Calderón candidato independiente, frente al de Ivonne Álvarez y Felipe de Jesús candidatos del PRI y PAN (Ver Tabla 5).

\section{Encuadres de candidatos en televisión frente a prensa escrita}

En el encuadre de juego estratégico en televisión no se obtuvieron diferencias estadísticas significativas, $F(2,234)=0.398, p=.672$, siendo Jaime Rodríguez quien tendía a presentar más este encuadre en su cobertura $(M=.25, D E=.29)$, seguido de Ivonne Álvarez $(M=.22, D E=.25)$ y por último de Felipe de Jesús $(M=.21, D E=.28)$ (Ver Tabla 6).

En el encuadre de asunto en televisión sí hubo diferencias estadísticas significativas entre candidatos, $F(2,234)=3.958, p=.022$, siendo Ivonne Álvarez quien más noticias con frame de asunto presentó $(M=.23, D E=.22)$, seguida de Felipe de Jesús $(M=.17, D E=.22)$ y por último Jaime Rodríguez fue quien menos presenta noticias con frame de éste tipo $(M=.15, D E=.20)$.Obteniendo éstos resultados una vez más se determina la variación del tratamiento informativo ofrecido al candidato independiente frente a los partidos tradicionales lo cual es objetivo específico de la presente investigación.

El encuadre de conflicto en televisión no presentó diferencias estadísticas significativas, $F(2$, 234) $=1.064, p=.347$, siendo Jaime Rodríguez quien tendía a presentar ligeramente más noticias con frame de conflicto $(M=.13, D E=.24)$, seguido de Felipe de Jesús $(M=.12, D E=.24)$ y de, por último, Ivonne Álvarez que presentó menos noticias con frame de conflicto $(M=.08, D E=.17)$.

Por último, en lo que respecta a televisión el encuadre de debate político sí se obtuvieron diferencias estadísticamente significativas, $F(2,234)=3.342, p=.037$, siendo Jaime Rodríguez quien presenta este frame en las noticias $(M=.08, D E=.22)$, seguido de Felipe de Jesús $(M=.03, D E=$ .13 ) con una presencia igual que Ivonne Álvarez $(M=.03, D E=.10)$. A diferencia del encuadre de asunto en el cual también se obtienen resultados estadísticamente significativos, Jaime Rodríguez Calderón es quien más presenta este encuadre en sus noticias frente a Ivonne Álvarez y Felipe de Jesús Cantú, candidatos de partidos (Ver Tabla 6). 
Tabla 6. Encuadres de candidatos en televisión

\begin{tabular}{|c|c|c|c|c|}
\hline $\begin{array}{l}\text { Medio de comu- } \\
\text { nicación }\end{array}$ & & Candidato & $M$ & $\overline{D E}$ \\
\hline \multirow{16}{*}{ Televisión } & \multirow[t]{4}{*}{$\begin{array}{l}\text { Encuadre de } \\
\text { juego estratégico }\end{array}$} & $\begin{array}{l}\text { Felipe de Jesús Cantú Rodrí- } \\
\text { guez (PAN) }\end{array}$ & .21 & .28 \\
\hline & & $\begin{array}{l}\text { Ivonne Liliana Álvarez García } \\
\text { (Alianza por tu seguridad) }\end{array}$ & .22 & .27 \\
\hline & & $\begin{array}{c}\text { Jaime Rodríguez Calderón el } \\
\text { Bronco }\end{array}$ & .25 & .29 \\
\hline & & Total & .23 & .28 \\
\hline & \multirow[t]{4}{*}{$\begin{array}{l}\text { Encuadre de } \\
\text { asunto }\end{array}$} & $\begin{array}{l}\text { Felipe de Jesús Cantú Rodrí- } \\
\text { guez (PAN) }\end{array}$ & .17 & .22 \\
\hline & & $\begin{array}{l}\text { Ivonne Liliana Álvarez García } \\
\text { (Alianza por tu seguridad) }\end{array}$ & .23 & .22 \\
\hline & & $\begin{array}{c}\text { Jaime Rodríguez Calderón el } \\
\text { Bronco }\end{array}$ & .15 & .20 \\
\hline & & Total & .23 & .28 \\
\hline & \multirow[t]{4}{*}{$\begin{array}{l}\text { Encuadre de con- } \\
\text { flicto }\end{array}$} & $\begin{array}{l}\text { Felipe de Jesús Cantú Rodrí- } \\
\text { guez (PAN) }\end{array}$ & .12 & .24 \\
\hline & & $\begin{array}{l}\text { Ivonne Liliana Álvarez García } \\
\text { (Alianza por tu seguridad) }\end{array}$ & .08 & .17 \\
\hline & & $\begin{array}{c}\text { Jaime Rodríguez Calderón el } \\
\text { Bronco }\end{array}$ & .13 & .24 \\
\hline & & Total & .11 & .22 \\
\hline & \multirow[t]{4}{*}{$\begin{array}{l}\text { Encuadre de de- } \\
\text { bate político }\end{array}$} & $\begin{array}{l}\text { Felipe de Jesús Cantú Rodrí- } \\
\text { guez (PAN) }\end{array}$ & .03 & .13 \\
\hline & & $\begin{array}{l}\text { Ivonne Liliana Álvarez García } \\
\text { (Alianza por tu seguridad) }\end{array}$ & .03 & .10 \\
\hline & & $\begin{array}{c}\text { Jaime Rodríguez Calderón el } \\
\text { Bronco }\end{array}$ & .08 & .22 \\
\hline & & Total & .05 & .16 \\
\hline
\end{tabular}

En la prensa escrita el uso de encuadre de juego estratégic ono se obtuvieron diferencias estadísticas significativas, $F(2,190)=0.981, p=.377$, siendo Felipe de Jesús quien tendía a presentar ligeramente más este encuadre en su cobertura $(M=.13, D E=.19)$, seguido de Ivonne Liliana $(M=$ $.11, D E=.15)$ y por último Jaime Rodríguez, que fue quien menos presentaba este encuadre $(M=.09$, $D E=.15$ ). A pesar de que los resultados no presentaron diferencias estadísticamente significativas, se puede observar que Jaime Rodríguez Calderón, único candidato independiente, es quien menos presentó este encuadre frente a los otros dos candidatos (Ver Tabla 7). 
Tabla 7. Encuadres de candidatos en prensa escrita

\begin{tabular}{|c|c|c|c|c|}
\hline $\begin{array}{l}\text { Medio de comu- } \\
\text { nicación }\end{array}$ & & Candidato & $M$ & $D E$ \\
\hline \multirow{16}{*}{ Prensa } & \multirow[t]{4}{*}{$\begin{array}{l}\text { Encuadre de } \\
\text { juego estratégico }\end{array}$} & $\begin{array}{l}\text { Felipe de Jesús Cantú Rodrí- } \\
\text { guez (PAN) }\end{array}$ & .13 & .19 \\
\hline & & $\begin{array}{l}\text { Ivonne Liliana Álvarez García } \\
\text { (Alianza por tu seguridad) }\end{array}$ & .11 & .15 \\
\hline & & $\begin{array}{c}\text { Jaime Rodríguez Calderón el } \\
\text { Bronco }\end{array}$ & .09 & .15 \\
\hline & & Total & .11 & .16 \\
\hline & \multirow[t]{4}{*}{$\begin{array}{l}\text { Encuadre de } \\
\text { asunto }\end{array}$} & $\begin{array}{l}\text { Felipe de Jesús Cantú Rodrí- } \\
\text { guez (PAN) }\end{array}$ & .17 & .20 \\
\hline & & $\begin{array}{l}\text { Ivonne Liliana Álvarez García } \\
\text { (Alianza por tu seguridad) }\end{array}$ & .15 & .18 \\
\hline & & $\begin{array}{c}\text { Jaime Rodríguez Calderón el } \\
\text { Bronco }\end{array}$ & .15 & .21 \\
\hline & & Total & .16 & .19 \\
\hline & \multirow[t]{4}{*}{$\begin{array}{l}\text { Encuadre de con- } \\
\text { flicto }\end{array}$} & $\begin{array}{l}\text { Felipe de Jesús Cantú Rodrí- } \\
\text { guez (PAN) }\end{array}$ & .11 & .20 \\
\hline & & $\begin{array}{l}\text { Ivonne Liliana Álvarez García } \\
\text { (Alianza por tu seguridad) }\end{array}$ & .06 & .13 \\
\hline & & $\begin{array}{c}\text { Jaime Rodríguez Calderón el } \\
\text { Bronco }\end{array}$ & .31 & .26 \\
\hline & & Total & .14 & .22 \\
\hline & \multirow[t]{4}{*}{$\begin{array}{l}\text { Encuadre de de- } \\
\text { bate político }\end{array}$} & $\begin{array}{l}\text { Felipe de Jesús Cantú Rodrí- } \\
\text { guez (PAN) }\end{array}$ & .05 & .17 \\
\hline & & $\begin{array}{l}\text { Ivonne Liliana Álvarez García } \\
\text { (Alianza por tu seguridad) }\end{array}$ & .04 & .11 \\
\hline & & $\begin{array}{c}\text { Jaime Rodríguez Calderón el } \\
\text { Bronco }\end{array}$ & .04 & .14 \\
\hline & & Total & .04 & .14 \\
\hline
\end{tabular}

El uso de encuadre de asunto en prensa no presentó diferencias estadísticamente significativas entre candidatos, $F(2,190)=0.257, p=.773$. En este caso fue Felipe de Jesús quien ligeramente presentó más este frame en su cobertura $(M=.17, D E=.20)$, seguido de Jaime Rodríguez $(M=.15$, $D E=.21)$ y por último Ivonne Álvarez, que fue quien menos presenta este encuadre en su cobertura $(M=.15, D E=.18)($ Ver Tabla 7$)$. 
En el uso de encuadre conflicto en prensa sí hubo diferencias estadísticas significativas entre candidatos, $F(2,190)=26.720, p<$.001. Jaime Rodríguez fue quien más presentó este encuadre en su cobertura $(M=.31, D E=.26)$, mientras que Felipe de Jesús presentó menos el encuadre de conflicto $(M=.11, D E .20)$ seguido de Ivonne Álvarez $(M=.06, D E=.13)$. El presente encuadre (de conflicto) es el único que en lo que respecta a prensa escrita arrojó diferencias estadísticas significativas, siendo el candidato independiente quien más presenta el mencionado encuadre en sus noticias frente a los candidatos de partidos.

Por último, el uso de encuadre de juego debate político en prensa no arrojó diferencias estadísticamente significativas entre candidatos, $F(2,190)=0.119, p=.888$, siendo Felipe de Jesús quien ligeramente más presentó este frame $(M=.05, D E=.17)$, seguido de Jaime Rodríguez $(M=.04, D E$ $=.14)$ y por último Ivonne Liliana $(M=.04, D E=.11)($ Ver Tabla 7$)$.

Figura 1. Carácter evaluativo de cada candidato en las noticias

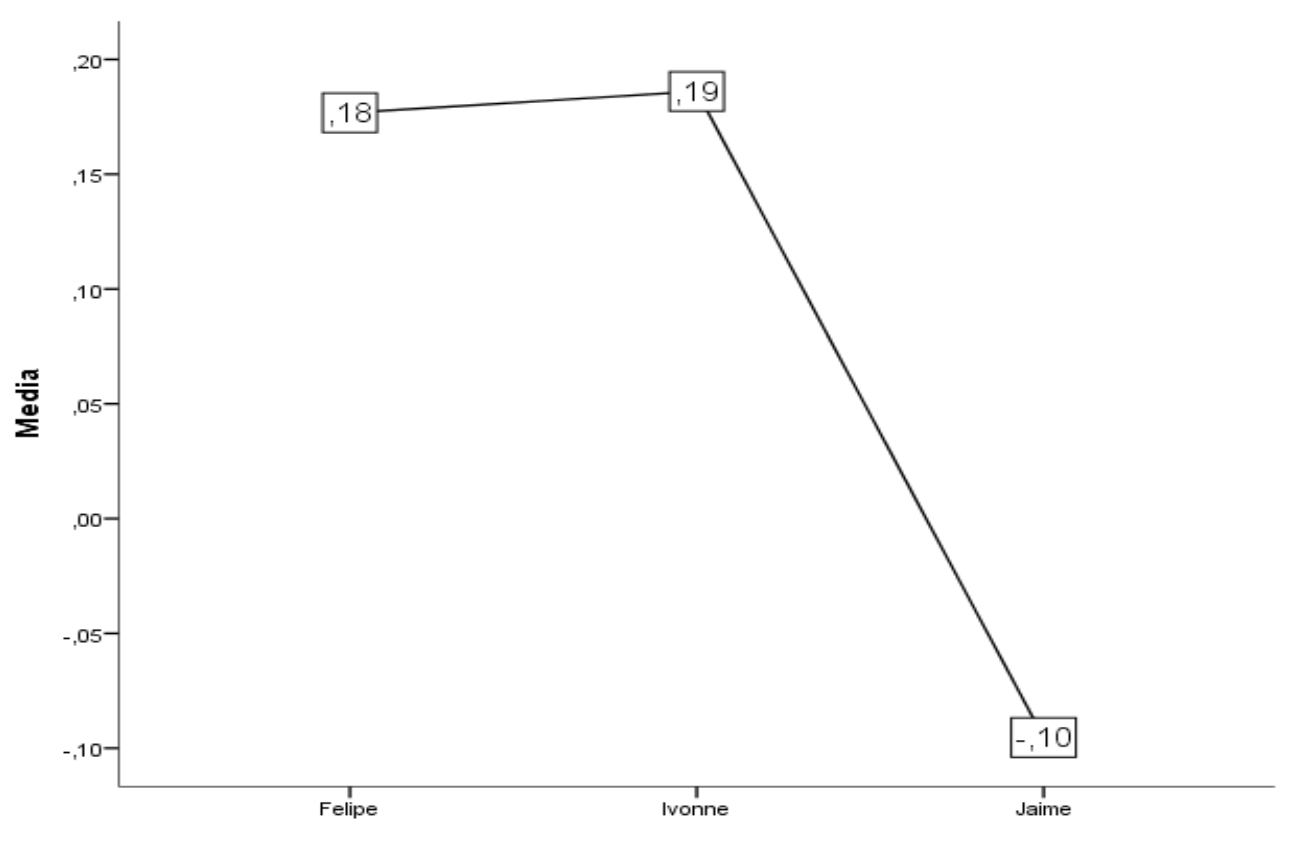

\section{Carácter evaluativo de los candidatos en las noticias}

El carácter evaluativo del candidato, es decir la valencia afectiva (positiva vs. negativa) que fue ofrecida a los candidatos en la cobertura realizada por los medios de la campaña electoral en sus noticias, presentó una diferencia estadísticamente significativa entre los candidatos a la gubernatura, $F(2,835)=49.782, p<.001$. El presente estudio arrojó que Jaime Rodríguez Calderón, candidato independiente a la gubernatura por el estado de Nuevo León, fue quien recibió un carácter evaluativo más desfavorable $(M=-.10)$, frente a Ivonne Álvarez candidata del PRI $(M=.19)$ y Felipe de Jesús 
candidato del PAN $(M=.18)$ que presentaron una valencia afectiva en sus noticias más positiva en contraposición (Ver Figura 1).

\section{Reflexiones finales}

Como se pudo observar y analizar en la teoría, dentro del proceso de las campañas electorales son los medios de comunicación quienes juegan un papel sumamente importante ya que los mismos dan a conocer a los candidatos y partidos políticos, sus propuestas y promesas de campaña, proyectos a desarrollar en beneficio de la sociedad e incluso aspectos de la vida personal de los mismos. Por ello es clara la importancia de conocer el contenido real al que los receptores están expuestos; esto a través de la cobertura informativa la que a su vez lleva consigo un tratamiento informativo o framing (Muñiz, 2015).

Es imposible dejar de lado la importancia y el poder que tienen los medios sobre la política y más en periodos de campañas electorales; dentro de las mismas se tiene una considerable presencia de los medios los cuales conllevan a un proceso de comunicación social fuerte de información política a la ciudadanía, siendo los medios quien jueguen un papel crucial transmitiendo información suministrada por la prensa escrita, radio, televisión etc. (Aceves, 2000).

En este estudio se pudo encontrar que los medios de comunicación, si bien no ofrecieron tratamiento informativo igual, si al menos presentaron una cobertura similar entre los candidatos poniéndolos en un rango equitativo de cobertura tanto para televisión como para la prensa escrita.

Esto hace reflejar lo que señala Dell'Oro (2014) con respecto a la cobertura que ofrecen los medios de comunicación a los candidatos en periodos de campañas electorales, haciéndolos protagonistas y entendiendo la campaña como un escenario en el que se encuentran los candidatos dando a conocer sus propuestas, trayectoria política, personalidad (pp. 132-134). Recordando que los resultados no fueron estadísticamente significativos, se pudo observar que lo que buscan los medios es dar la cobertura a los candidatos; al menos en el caso del presente estudio no hubo diferenciación significativa en la cobertura informativa, siendo Ivonne Álvarez quien encabezó los resultados en televisión, siguiendo Jaime Rodríguez el Bronco y por último Felipe de Jesús Cantú. En el caso de la prensa escrita, Ivonne Álvarez siguió encabezando la lista, pero esta vez Felipe de Jesús Cantú obtuvo mayor cobertura que Jaime Rodríguez candidato independiente. Sin embargo, como se menciona anteriormente, los medios ofrecieron una cobertura similar a Jaime frente a los candidatos de partidos tradicionales.

Acerca del tratamiento informativo o framing que los medios de comunicación dieron a Jaime Rodríguez Calderón, fue similar al ofrecido a Ivonne Álvarez y Felipe de Jesús Cantú. En todo 
caso, se pudo observar según las pruebas correspondientes aplicadas que en el caso de encuadre estratégico se presentó un tratamiento informativo similar, siendo Jaime Rodríguez quien más presentó el encuadre ante los medios. Por otra parte en el encuadre de asunto y de debate, se pudo observar que al igual que en el encuadre estratégico los medios ofrecieron un tratamiento informativo similar, ésta vez siendo Ivonne Álvarez quien más presentó el encuadre de asunto y Jaime Rodríguez el de debate. En el caso del encuadre de conflicto no sucedió lo mismo ya que Jaime Rodríguez presentó un mayor número de noticias con este encuadre.

Con lo anterior recordamos lo que dice Sadaba (2001) acerca de la forma en la que los medios utilizan los frames o encuadres dando una crítica objetiva de la realidad. Dicha cobertura que brinda la televisión y prensa escrita a las campañas electorales se encuadran en los términos antes mencionados los cuales arrojaron resultados a los objetivos principales de la presente investigación (juego estratégico, asunto, conflicto y debate), lo cual permitió demostrar que a pesar de haber diferencias entre el tratamiento informativo que se le ofreció a un candidato frente a otras dichas diferencias no fueron estadísticamente significativas (Gerth \& Siegert, 2012). También fue necesario determinar si se encontraron diferencias en el tratamiento informativo que televisión ofreció a los candidatos a la gubernatura del estado de Nuevo León, frente a la prensa escrita.

Con lo mencionado anteriormente, se pudo observar en los resultados obtenidos que se presentaron resultados estadísticamente significativos en televisión frente a prensa escrita, dichas variaciones estadísticamente significativas se presentaron dentro del mismo medio (en los distintos encuadres como juego estratégico, debate político, conflicto). Con ello cual podemos observar que sí hubo una variación del tratamiento informativo ofrecido por la televisión frente a la prensa en los tres candidatos a la gubernatura. Con esto podemos retomar lo señalado acerca del entorno político, en momentos de campañas electorales se intensifica el trabajo de los medios de comunicación y los candidatos, así como de las élites para tratar de introducir sus encuadres en el tratamiento informativo (Hanggli \& Kriesi, 2012; Muñiz, 2015).

En el caso de la valencia afectiva (positiva vs. negativa) que los medios de comunicación ofrecieron al candidato independiente frente a los partidos tradicionales, se observó que Jaime Rodríguez Calderón, candidato independiente fue quien más recibió un carácter negativo desfavorable frente los otros candidatos que contendían por la gubernatura del estado.

$\mathrm{Al}$ igual que en los encuadres, no solamente fue necesario determinar el carácter evaluativo con el que se presentó a los candidatos sino también determinar la valencia afectiva ofrecida a los mismos (positiva vs. negativa) que la televisión ofreció a los candidatos frente al que ofreció la prensa escrita, permitiendo así determinar de acuerdo a las pruebas realizadas que sí hubo una variación considerable. En el caso de la prensa escrita, el candidato del PAN fue quien más carácter positivo presentó frente a la televisión; por otra parte, a pesar de que la candidata del PRI no presentó una variación considerable entre ambos medios al igual que el candidato del PAN fue la prensa escrita la 
que le dio una evaluación mayormente positiva frente a la televisión. Por último, se pudo observar que el candidato independiente presento un carácter desfavorable en ambos medios, con lo que se puede afirmar y responder que efectivamente los medios ofrecieron una valencia afectiva distinta al candidato independiente frente al de los partidos tradicionales, siendo el primero quien resulto expuesto con un carácter desfavorable ante ambos medios.

En este sentido, los resultados nos llevan a retomar lo señalado por Lozano et al. (2012), acerca de cómo los medios informativos detallan y dan una cobertura electoral que en gran parte se dedica al desprestigio entre un partido y/o un político y otro, lo cual permite descalificarlos o bien posicionarlos de manera positiva o negativa ante los medios de comunicación, pero aplicándolo en este caso a la cobertura de la campaña electoral de Nuevo León de 2015 en este caso prensa escrita y televisión.

\section{Agradecimientos}

Agradezco al Dr. Carlos Muñiz, quien fue clave para la elaboración de dicho proyecto. Gracias a mis padres por su apoyo incondicional.

\section{Declaración de conflicto de intereses}

La autora no informó ningún posible conflicto de intereses.

\section{Fondos}

Este artículo es realizado gracias al apoyo del Consejo Nacional de Ciencia y Tecnología (CONACyT) para la realización de los estudios de maestría, así como partir de los datos obtenidos en el proyecto de investigación titulado "Análisis de la cobertura mediática de la campaña electoral 2015 a gobernador de Nuevo León en la prensa y televisión locales”, financiado por la UANL dentro del Programa de Apoyo a la Investigación Científica y Tecnológica PAICYT-2015 (Clave CSH012-15). Este texto forma parte de la tesis de maestría. 


\section{Referencias}

Aalberg, T., Strömbäck, J., \& de Vreese, C. H. (2012). The framing of politics as strategy and game: A review of concepts, operationalizations and key findings. Journalism, 13(2), 162-178. https://doi.org/10.1177/1464884911427799

Aceves, F. (2000). La investigación académica sobre el papel de los medios de comunicación en los procesos electorales en México. Comunicación y Sociedad, (37), 11-36.

Aceves, F. (2004). Monitoreo de medios y democratización en América Latina. La participación ciudadana en la vigilancia de la función informativa de los medios de comunicación de masas. Comunicación y Sociedad, (1), 91-108.

Acuerdo CEE (2015). Acuerdo del Consejo General de la Comisión Estatal Electoral, relativo a determinar los límites a las aportaciones de carácter privado realizadas a los candidatos independientes durante el proceso electoral 2014-2015. Acuerdo CEE/CG/121/2015, derivado del acuerdo INE/CG305/2015. http://www.cee.nl.org.mx/sesiones/2013_2015/acuerdos/20150527.pdf

Ahuactzin, C. E., Ríos, C. I. \& Cisneros, J. (2014). Framing y significado del spot televisivo electoral de Enrique Peña Nieto en la campaña presidencial. En J. A. Meyer (Coord.), Comunicación política y elecciones federales en México (pp. 210-237). Comunicación Social.

Bateson, G. (1972) Steps to an ecology of mind: Collected essays in anthropology, psychiatry, evolution and epistemology. Ballantine Books.

Berumen, G., \& Medellín L. (2016). Marketing de los candidatos a la gubernatura de Nuevo León en las redes sociales durante el proceso electoral de 2015. Apuntes Electorales, (54), 55-90.

Cámara de Diputados del H. Congreso de La Unión. (2020, 13 de abril). Ley General de Instituciones y Procesos Electorales. Diario Oficial de la Federación. http://www.diputados.gob.mx/LeyesBiblio/pdf/LGIPE_130420.pdf

Cappella, J., \& Jamieson, K. (1997). Spiral of cynicism. The press and the public good. Oxford University Press.

Comisión Estatal Electoral Nuevo León (2015). Resultados del cómputo estatal 2015. http://ceeresultadosweb.azurewebsites.net/index.html

de Vreese, C. H. (2012). New avenues for framing research. American Behavioral Scientist, 56(3), 365-375. https://doi.org/10.1177/0002764211426331

Dell'Oro, J. (2014). Credibilidad, experiencia y honestidad.c் Son suficientes para ser un buen candidato? Diálogo Político, 31(1), 69-79.

Dimitrova, D. V., \& Strömbäck, J. (2012). Election news in Sweden and the United States: A comparative study of sources and media frames. Journalism, 13(5), 604-619. https://doi.org/10.1177/1464884911431546

Elenbaas, M., \& de Vreese, C. H. (2008). The effects of strategic news on political cynicism and vote choice among young voters. Journal of Communication, 58(3), 550-567. https://doi.org/10.1111/j.1460-2466.2008.00399.x

Entman, R. (1991). Framing U.S. coverage of international news: Contrast in narratives of the KAL and Iran air incidents. Journal of Communication, 41(4), 6-27. https://doi.org/10.1111/j.1460-2466.1991.tbo2328.x

Entman, R. (1993). Framing: Toward clarification of a fractured paradigm. Journal of Communication, 43(4), 51-58. https://doi.org/10.1111/j.1460-2466.1993.tbo1304.x 
Gerth, M. A., \& Siegert, G. (2012). Patterns of consistence and constriction: How news media frame the coverage of direct democratic campaigns. American Behavioral Scientist, 56(3), 279-299. https://doi.org/10.1177/0002764211426326

Giménez P. (2006). Una nueva visión del proceso comunicativo: La teoría del enfoque (Framing). Comunicación y Hombre, (2), 55-66.

Gitlin, T. (1980). The whole world is watching. University of California Press.

Goffman, E. (1986). Frame Analysis. Los marcos de la experiencia. Siglo XXI.

Hanggli, R., \& Kriesi, H. (2012). Frame construction and frame promotion (strategic framing choices). American Behavioral Scientist, 56(3), 260-278. https://doi.org/10.1177/0002764211426325

Igartua, J. J., Muñiz, C, \& Otero, J. A. (2005). El tratamiento informativo de la inmigración en la prensa y la televisión española. Una aproximación empírica desde la teoría del Framing. Global Media Journal, 3(5), 1-15.

Lozano, J. C., Cantú, J., Martínez, F. J., \& Smith, C. (2012). Evaluación del desempeño de los medios informativos en las elecciones de 2009 en Monterrey. Comunicación y Sociedad, (18), 173-197.

Marañón, F (2015). El spot como herramienta de persuasión política. Análisis del impacto de la publicidad política en la desafección política a través de la ruta central y periférica [Tesis de doctorado, Universidad Autónoma de Nuevo León]. Repositorio Académico Digital. http://eprints.uanl.mx/10985/

Martínez, F. (2008). ¿Por qué importan las campañas electorales? Centro de Investigaciones Sociológicas.

Martínez, F., \& Godínez, F. (2013). La agenda de los telediarios en la contienda del 2012. Derecho a Comunicar, (7), 59-75. https://doi.org/10.13140/2.1.2994.1444

Matthes, J. (2012). Framing politics: An integrative approach. American Behavioral Scientist, 56(3), 247-259. https://doi.org/10.1177/0002764211426324

McCombs, M., \& Evatt. D. (1995). Los temas y los aspectos: explorando una nueva dimensión de la agenda setting. Comunicación y Sociedad, 8(1), 7-32.

Meyer, J. A. (2014). Comunicación Política y elecciones federales en México. Comunicación Social.

Muñiz, C. (2011). Encuadres noticiosos sobre migración en la prensa digital mexicana. Un análisis de contenido exploratorio desde la teoría del framing. Convergencia, 18(55), 213-239.

Muñiz, C. (2015). La política como debate temático o estratégico framing de la campaña electoral mexicana de 2012 en la prensa digital. Comunicación y Sociedad, (23), 67-69. https://doi.org/10.32870/cys.voi23.64

Muñiz C., \& Ramírez, J. (2014). Framing de la violencia e inseguridad en México. Los empresarios frente al narcotráfico en México. Tratamiento informativo de las reacciones empresariales ante situaciones de violencia e inseguridad. Estudios sobre el Mensaje Periodístico, 21(1), 437-453. https://doi.org/10.5209/rev_ESMP.2015.v21.n1.49104

Rospir, J. (2003). Introducción a la comunicación política. En S. Berrocal (Coord.), Comunicación política en televisión y nuevos medios (pp. 21-54). Ariel.

Ryan, P. (2012). Agenda setting in English Canada in the age of minority government, 20042011 [Tesis doctoral no publicada]. Ryerson University/York University, Toronto, Canadá.

Sádaba, T. (2001). Origen, aplicación y límites de la "teoría del encuadre” (Framing) en comunicación. Comunicación y sociedad, 14 (2), 143-145. 
Scheufele, D. (2000) Agenda-setting, priming and framing revisited: another look at cognitive effects of political communication. Mass Communication and Society, 3(2-3), 297-316. https://doi.org/10.1207/S15327825MCSo323_07

Schuck, A., Boomgaarden, H. G., \& de Vreese, C. H. (2013). Cynics all around? The impact of election news on political cynicism in comparative perspective. Journal of Communication, 63(2), 287-311. https://doi.org/10.1111/jcom.12023

Semetko, H., \& Valkenburg, P. (2000). Framing European politics: A content analysis of press and television news. Journal of Communication, 5O(2), 93-109. https://doi.org/10.1111/j.14602466.2000.tbo2843.x

Soler, M. (2001). Campañas electorales y democracia en España. Publicacions de la Universitat Jaume I.

Tankard, J. (1991, agosto). Media frames: Approaches to conceptualization and measurement [Ponencia]. Journalism and Mass Communication Convention, Boston, Estados Unidos.

Toussaint, F. (2000). Las campañas electorales del 200 en televisión. El caso mexicano. Revista Mexicana de Ciencias Políticas, 44(180), 39-56.

Valdez, A. (2010). Las campañas electorales en la nueva sociedad de información y el conocimiento, Estudios Políticos, (20), 155-165. 